\title{
Evaluation of cone function by a handheld non-mydriatic flicker electroretinogram device
}

\author{
This article was published in the following Dove Press journal: \\ Clinical Ophthalmology \\ 30 June 2016 \\ Number of times this article has been viewed
}

\section{Natsuko Nakamura' \\ Kaoru Fujinami' \\ Yoshinobu Mizuno² \\ Toru Noda ${ }^{2}$ \\ Kazushige Tsunoda'}

'Laboratory of Visual Physiology, Division of Vision Research, National Institute of Sensory Organs, ${ }^{2}$ Department of Ophthalmology, National Hospital Organization, National Tokyo Medical Center, Tokyo, Japan
Correspondence: Kazushige Tsunoda Laboratory of Visual Physiology, Division of Vision Research, National Institute of Sensory Organs, 2-5-I Higashigaoka, Meguro-ku, Tokyo I52-8902, Japan $\mathrm{Tel}+8|334| \mathrm{l} 01 \mid \mathrm{I}$ ext 66 I5

Fax $+8|334| I 0185$

Email tsunodakazushige@kankakuki.go.jp
Purpose: Full-field electroretinograms (ERGs) are used to evaluate retinal function in patients with various types of hereditary and acquired retinal diseases. However, ERG recordings require relatively invasive procedures, including pupillary dilation and the use of contact lens electrodes. Thus, it would be helpful to have a simpler and noninvasive screening method. The purpose of this study was to determine whether a new, handheld, portable ERG device, RETeval ${ }^{\mathrm{TM}}$, can be used to screen patients for cone dysfunction.

Patients and methods: Thirty-five eyes of 35 patients who had reduced cone responses ascertained by a conventional ERG system using contact lens electrodes were studied. The causative diseases included achromatopsia, cone dystrophy, cone-rod dystrophy, retinitis pigmentosa, choroidal dystrophy, autoimmune retinopathy, and Stargardt disease. The flicker ERGs were recorded with the RETeval ${ }^{\mathrm{TM}}$ under undilated conditions with skin electrodes (stimulus strength, $3.0 \mathrm{~cd} \cdot \mathrm{s} / \mathrm{m}^{2}$; frequency, $28.3 \mathrm{~Hz}$ ), and the responses were compared to that of 50 healthy eyes. The amplitudes and implicit times of the fundamental component of the flicker ERGs were analyzed in three age groups: Group A, $\leq 20$ years; Group B, 21-40 years; and Group $\mathrm{C}, \geq 41$ years.

Results: In all of the age groups, the amplitudes of the ERGs were significantly smaller and the implicit times significantly longer in patients with cone dysfunction than in the control eyes. All but one of the patients had flicker amplitudes lower than the mean -2.0 standard deviation of control eyes.

Conclusion: The RETeval ${ }^{\mathrm{TM}}$ has a potential of being used to screen for cone dysfunction. The entire examination takes $<5$ minutes and does not require pupil dilatation or a contact lens electrode. Although the flicker responses do not provide information on the scotopic functions, the RETeval ${ }^{\text {TM }}$ device can be used to determine which patients require additional full-field ERG testing with dilated pupils under both scotopic and photopic conditions.

Keywords: RETeval ${ }^{\mathrm{TM}}$, flicker ERG, cone dysfunction, retina

\section{Introduction}

Full-field electroretinograms (ERGs) recorded with a contact lens electrode are generally used to evaluate retinal function. Among the six types of responses elicited by different stimulus intensities under different states of adaptation, ${ }^{1}$ the light-adapted $30 \mathrm{~Hz}$ flicker ERGs have been used to assess cone function not only in patients with hereditary retinal diseases, such as cone-rod dystrophy, achromatopsia, ${ }^{2}$ and retinitis pigmentosa, ${ }^{3,4}$ but also in those with acquired disorders, such as acute zonal occult outer retinopathy, ${ }^{5}$ paraneoplastic and autoimmune retinopathies, ${ }^{6}$ diabetic retinopathy, ${ }^{7,8}$ central retinal vein occlusion, ${ }^{9,10}$ and drug toxicities. ${ }^{11,12}$ However, ERG recordings cannot be easily performed on young children except in the clinics with high expertise, where skin electrodes or Dawson-Trick-Litzkow (DTL) electrodes are routinely used. ${ }^{13}$ 
Moreover, for some retinal diseases that do not have distinct ophthalmoscopic abnormalities, ${ }^{14-16}$ ERG testing may occasionally not be performed because the recording procedures are more complex than other ophthalmological tests and need to be performed with special equipments in an electrically shielded examination room. Thus, it would be helpful to have a simpler and noninvasive recording method for the screening of patients suspected of having cone dysfunction.

The RETevalTM system (LKC Technologies, Inc., Gaithersburg, MD, USA) is a new handheld, portable ERG device that can record $28.3 \mathrm{~Hz}$ flicker ERGs with adhesive skin electrodes under non-mydriatic conditions. ${ }^{17}$ This system was originally designed to screen for retinal ischemia due to diabetic retinopathy and monitor vigabatrin toxicity. ${ }^{7,8,11}$ However, it has a potential of evaluating the cone function in different disease conditions. ${ }^{18}$ We have recorded flicker ERGs with the RETeval ${ }^{\mathrm{TM}}$ system from patients with various types of retinal disorders to determine whether this can be used to screen for cone dysfunction.

\section{Materials and methods}

The inclusion criteria were patients who had reduced $30 \mathrm{~Hz}$ flicker ERG responses recorded with a conventional ERG system using a contact lens electrode. The amplitudes and implicit times of the ERGs recorded with the RETeval ${ }^{\mathrm{TM}}$ system were compared to those recorded from control eyes.

Thirty-five eyes of 35 patients ( 18 males and 17 females) were studied. Their average age was $33.1 \pm 17.9$ years ( \pm standard deviation [SD]) with a range of 8-61 years. All of these patients had reduced $30 \mathrm{~Hz}$ flicker ERGs recorded with contract lens electrodes and conventional ERG systems (SG2002, LKC Technologies, Inc., and LE4000, Tomey Corporation, Aichi, Japan) that were lower than the mean -2.0 SD of normal eyes. The full-field ERGs were recorded in accordance with the protocol for ERGs by the International Society for Clinical Electrophysiology of Vision. ${ }^{1}$

The causative diseases included achromatopsia (six eyes), cone dystrophy (five eyes), cone-rod dystrophy (eight eyes), retinitis pigmentosa (12 eyes), choroidal dystrophy (one eye), autoimmune retinopathy (two eyes), and Stargardt disease (one eye). Flicker ERGs were recorded from these patients with cone dysfunction with the RETeval ${ }^{\mathrm{TM}}$ under undilated conditions with skin electrodes according to the preset protocol, that is, stimulus strength, $3.0 \mathrm{~cd} \cdot \mathrm{s} / \mathrm{m}^{2}$; frequency, $28.3 \mathrm{~Hz}$; sampling rate, 1,953 Hz; and background light, none. The $28.3 \mathrm{~Hz}$ frequency was chosen to be within International Society for Clinical Electrophysiology of Vision guidelines ${ }^{1}$ for recording $30 \mathrm{~Hz}$ flicker ERGs while avoiding an overlap of the harmonics of the stimulus frequency with the harmonics of the power line frequencies of 50 or $60 \mathrm{~Hz}$. The sampling rate $(1,953 \mathrm{~Hz})$ was an integer multiple of the stimulus frequency to aid in the Fourier analysis of the recordings. The data acquisition time was 5-10 seconds, which was dependent on a real-time estimate of the standard error of the mean for the implicit times. The right eyes of patients were tested, and the results of two consecutive trials were averaged and used for the statistical analyses. Flicker ERGs were also recorded from 50 healthy eyes consisting of 14 males and 36 females with the RETeval ${ }^{\mathrm{TM}}$. The average age of the control subjects was $28.5 \pm 13.0$ years with a range of 4-56 years. The pupil diameters were manually measured with a pupil gauge before the recordings with a background luminance of $30 \mathrm{~cd} / \mathrm{m}^{2}$. The pupil diameter at the time of the recordings was not significantly different between the patients $(4.3 \pm 0.7$ $\mathrm{mm}$ ) and controls ( $4.3 \pm 0.6 \mathrm{~mm} ; P=0.97$, Mann-Whitney $U$-test) (Figure S1). Although the RETeval ${ }^{\text {TM }}$ device has a built-in infrared camera that can track and measure the pupil size from the video images, this feature was not used in the protocol for this study. The pupil tracking software requires an unobstructed view of the pupil, which is difficult in some patients because the upper eyelids partially blocked the view of the pupil. The analysis of the flicker ERGs by the RETeval $^{\text {TM }}$ device is similar to the cycle-by-cycle analysis; ${ }^{19}$ therefore, only the reconstructed waveforms are shown. The reconstructed waveform uses the first eight harmonics because almost all of the energy in the responses is found in these harmonics. ${ }^{20}$ The fundamental component of the reconstructed waveform was used for the statistical analyses..$^{20,21}$ The amplitudes and implicit times of the eyes of patients were compared to those of the control eyes by Mann-Whitney $U$-tests.

This study was approved by the Ethics Committee of the National Institute of Sensory Organs (R15-006). Written informed consent was obtained from all patients. The procedures conformed to the tenets of the World Medical Association's Declaration of Helsinki.

\section{Results}

The mean amplitude of the flicker ERG was $2.5 \pm 3.4 \mu \mathrm{V}$ in the 35 eyes with cone dysfunction and $20.9 \pm 5.2 \mu \mathrm{V}$ in the 50 control eyes (Figure 1). The mean implicit time was $36.1 \pm 3.8 \mathrm{~ms}$ in 21 of the 35 eyes with cone dysfunction and $30.9 \pm 1.5 \mathrm{~ms}$ in the 50 control eyes. The implicit time could not be measured in 14 eyes with cone dysfunction because of the extremely low amplitudes of the flicker ERGs.

Because of the wide range in the ages of the patients, we divided them into three age groups; Group $\mathrm{A} \leq 20$ years, 


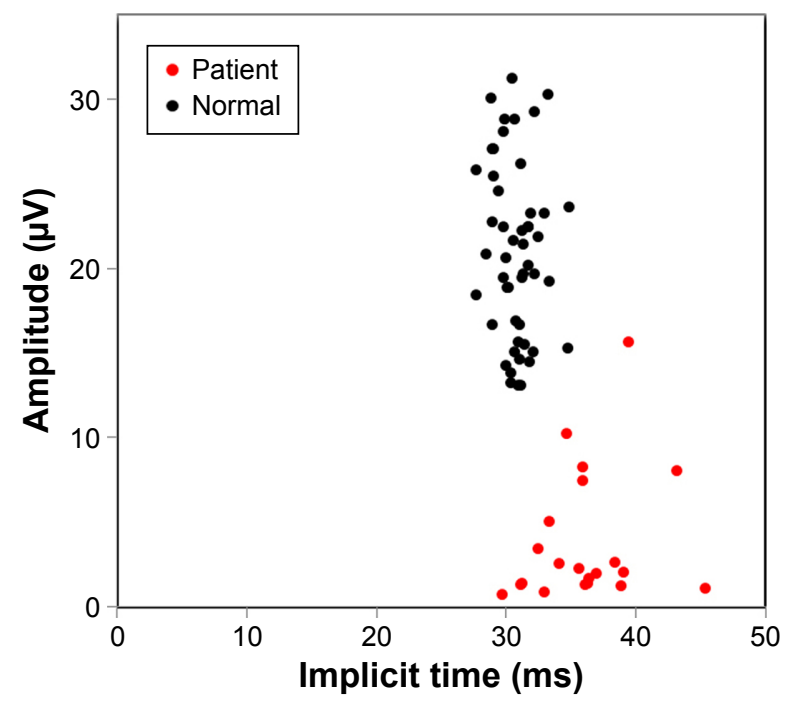

Figure I Amplitudes and implicit times of the ERGs recorded with the RETeval ${ }^{\mathrm{TM}}$ device.

Notes: Scatterplot of the amplitudes and implicit times of the RETeval ${ }^{\mathrm{TM}}$ ERGs recorded from patients with cone dysfunction (red) and controls (black). The implicit time was not measurable in 14 of 35 eyes due to extremely low amplitude because of the cone dysfunction.

Abbreviation: ERGs, electroretinograms.

Group B $21-40$ years, and Group C $\geq 41$ years. In all of the groups, the amplitudes and the implicit times were significantly different between patients and controls (Table 1). In addition, all of the patients had RETeval ${ }^{\text {TM }}$ flicker ERG amplitudes lower than the mean value $-2.0 \mathrm{SD}$ of the controls except for one patient with retinitis pigmentosa in Group C.

Scatterplots of the amplitudes and implicit times showing the correlations between the results obtained by the RETeval ${ }^{\mathrm{TM}}$ and conventional ERG system are shown in Figure 2. There was a significant positive correlation between two recording methods in the amplitudes ( $r=0.576$, $P<0.001, \mathrm{n}=35$; Pearson correlation coefficient). For the implicit times, on the other hand, the correlation was not significant $(r=-0.065, P=0.805, \mathrm{n}=17$; Pearson correlation coefficient). It should be noted that the RETeval ${ }^{\mathrm{TM}}$ and the conventional ERG were not recorded under the same conditions; all of the eyes were light-adapted before recording but the background light of the RETeval ${ }^{\mathrm{TM}}$ was off during the recording of 5-10 seconds to reduce the total light intensity so that patients with photophobia could keep fixating the target more steadily. In addition, the pupils were not dilated for the RETeval $^{\mathrm{TM}}$ but were dilated for the conventional ERGs.

A representative case of an 8-year-old boy who was diagnosed with cone-rod dystrophy is shown in Figure 3. The ophthalmoscopic appearance was normal, but the conventional photopic ERGs were extinguished, and the scotopic responses were markedly reduced (Figure $3 \mathrm{~A}$ and $\mathrm{B}$ ). The flicker responses were also extinguished in the RETeval ${ }^{\mathrm{TM}}$ (Figure 3C). The sinusoidal lines in red in Figure 3C represent the fundamental component of the RETeval ${ }^{\mathrm{TM}} \mathrm{ERGs}$, and the black lines indicate the reconstructed flicker ERG waveform, which is the combination of first eight harmonics (Figure 3C). In the patients, both the fundamental components and reconstructed waveforms are so low in amplitudes that they are merged into a single line.

Another example of a 4-year-old boy is shown in Figure 4. He had no symptoms, but his 7-year-old sister and father were diagnosed with retinitis pigmentosa. The ophthalmoscopic appearance of his fundus suggested that this boy was also affected with retinitis pigmentosa (Figure 4A), but he did not agree to have the ERG examination with the conventional system with contact lens electrodes. The flicker responses in the RETeval ${ }^{\mathrm{TM}}$ showed reduced amplitudes in both eyes (1.1 $\mu \mathrm{V}$, right eye and $0.56 \mu \mathrm{V}$, left eye), which were lower than the mean $-2.0 \mathrm{SD}$ of the control eyes (Figure 4B).

The ERGs shown in Figure 5A are from a 23-year-old male who has had low visual acuity and photophobia bilaterally

Table I The amplitudes and the implicit times of flicker responses in three age groups

\begin{tabular}{|c|c|c|c|c|c|c|c|c|c|}
\hline \multirow[t]{2}{*}{ Subjects } & \multicolumn{3}{|l|}{ Age } & \multicolumn{3}{|c|}{ Amplitude } & \multicolumn{3}{|c|}{ Implicit time } \\
\hline & Mean & SD & $P$-value* & Mean & SD & $P$-value* & Mean & SD & $P$-value* \\
\hline \multicolumn{10}{|c|}{ Group A ( $\leq 20$ years) } \\
\hline Patients $(n=12)$ & 13.2 & 4.02 & 0.058 & 0.99 & 1.00 & $<0.001$ & 34.3 & 3.49 & 0.028 \\
\hline Controls $(n=10)$ & 10.0 & 3.62 & & 18.7 & 4.00 & & 30.1 & $\mathrm{I} .34$ & \\
\hline \multicolumn{10}{|c|}{ Group B ( 2 I-40 years) } \\
\hline Patients $(\mathrm{n}=9)$ & 29.9 & 7.18 & 0.581 & 3.36 & 3.65 & $<0.001$ & 35.9 & 1.98 & $<0.001$ \\
\hline Controls $(n=30)$ & 27.9 & 4.05 & & 22.5 & 5.45 & & 30.9 & 1.32 & \\
\hline \multicolumn{10}{|l|}{ Group C ( $\geq 4$ I years) } \\
\hline Patients $(n=\mid 4)$ & 52.1 & 4.88 & 0.106 & 3.15 & 4.32 & $<0.00$ I & 37.0 & 4.53 & 0.002 \\
\hline Controls $(n=10)$ & 48.6 & 4.60 & & 18.5 & 3.69 & & 31.5 & 2.11 & \\
\hline
\end{tabular}

Note: *Mann-Whitney U-tests.

Abbreviation: SD, standard deviation. 

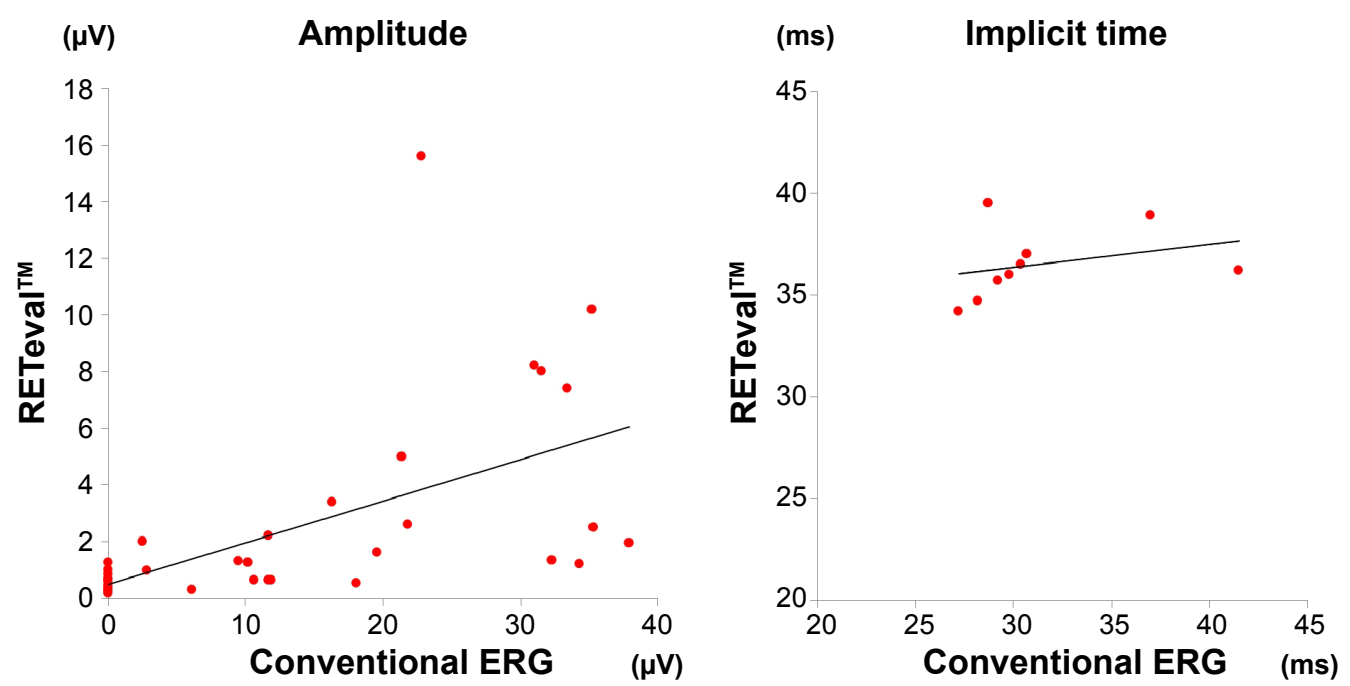

Figure 2 Scatterplots showing the relationship between the results obtained by the RETeval ${ }^{\mathrm{TM}}$ and conventional ERG in patients with cone dysfunction.

Notes: There was a significant positive correlation in the amplitude ( $r=0.576, P<0.00 \mathrm{I}, \mathrm{n}=35$; Pearson correlation coefficient). It should be noted that pupils were not dilated for the RETeval ${ }^{\mathrm{TM}}$ but were dilated for the conventional ERGs.

Abbreviation: ERGs, electroretinograms.

A
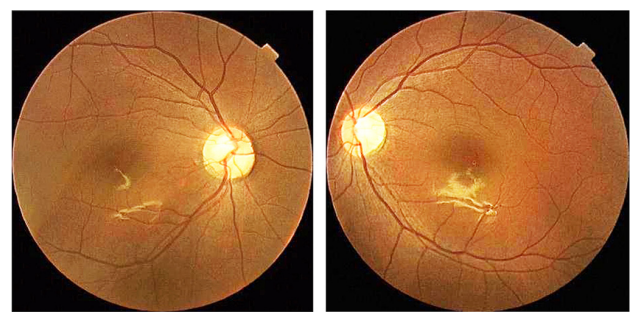

B

Patient

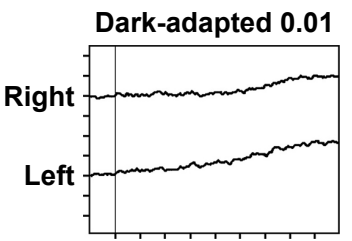

Dark-adapted 10.0
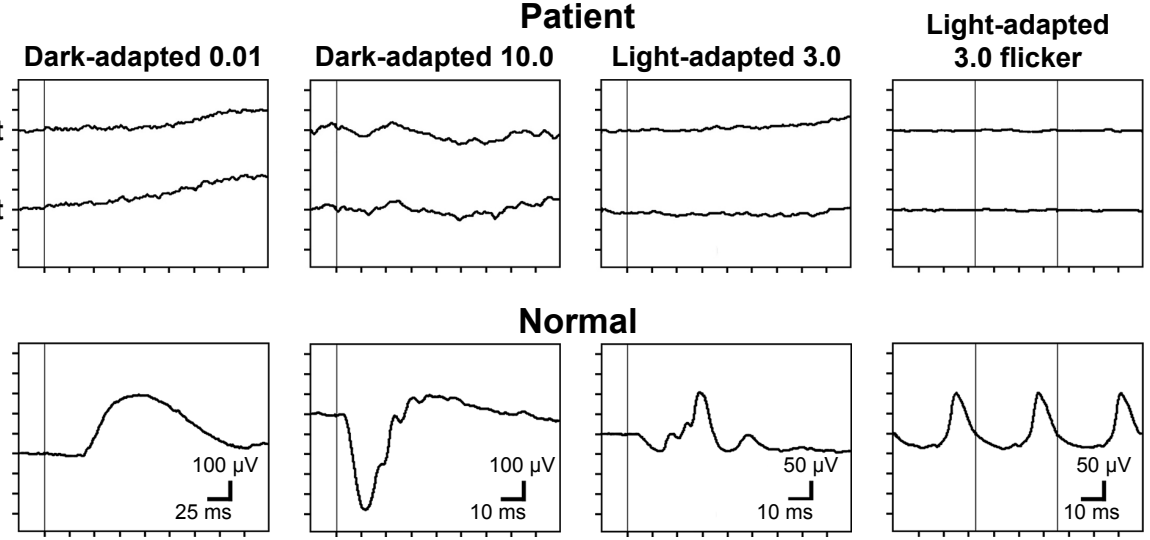

C

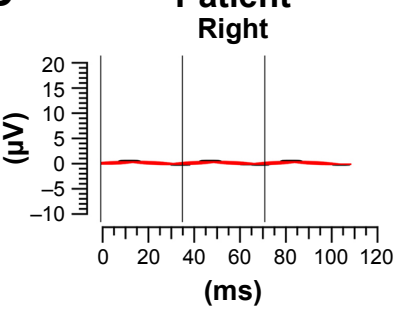

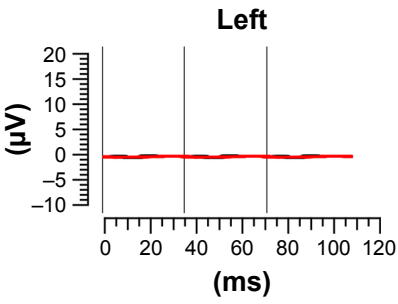

(ms)
Normal Right

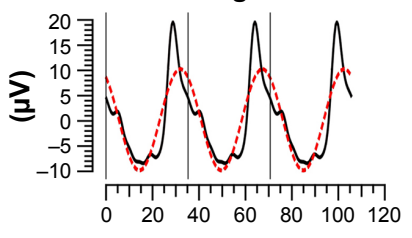

(ms)

Figure 3 An 8-year-old boy diagnosed with cone-rod dystrophy.

Notes: (A) Fundus photographs of the patient. (B) Conventional full-field ERGs: (top panel): Patient; (bottom panel): Normal subject. (C) RETeval TM ERGs from patient (left and middle) and control (right). The ophthalmoscopic appearance was normal, but flicker ERG responses were extinguished both in the conventional ERGs and the RETeval ${ }^{\mathrm{TM}}$ ERGs. The sinusoidal lines in red represent the fundamental component of the RETeval ${ }^{\mathrm{TM}}$ ERGs, and the black lines indicate the reconstructed flicker ERG waveform, which is the combination of first eight harmonics. The normal example of the RETeval ${ }^{T M}$ ERG was taken from a 7-year-old boy. The pupil diameters of the patient and normal subject are 5.0 and $4.0 \mathrm{~mm}$, respectively.

Abbreviation: ERGs, electroretinograms. 
A
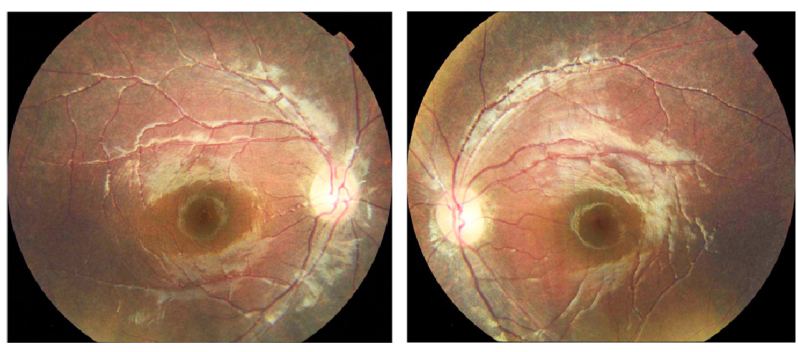

B

Patient

Right

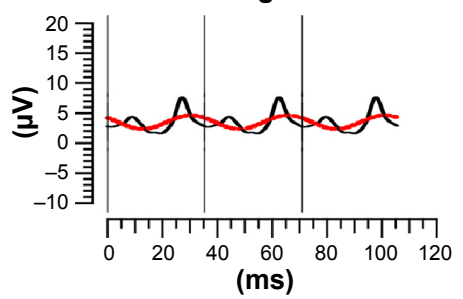

Left

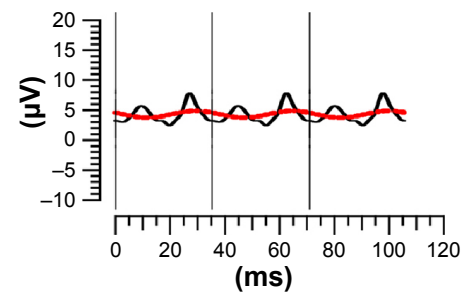

Normal

Right

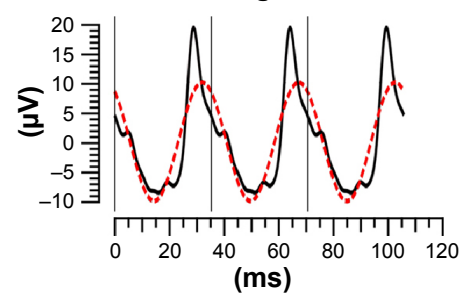

Figure 4 A 4-year-old boy whose older sister and father were diagnosed with retinitis pigmentosa.

Notes: (A) Fundus photographs of the patient. (B) RETeval TM ERGs from this patient (left and middle) and a control (right). The ophthalmoscopic appearance was normal, but the RETeval ${ }^{\mathrm{TM}}$ showed reduced amplitudes in both eyes; lower than the mean -2.0 standard deviations of normal eyes. This patient did not agree to have conventional ERG recordings with contact lens electrodes. The normal example of the RETeval ${ }^{\mathrm{TM}}$ ERG was taken from a 7-year-old boy. The pupil diameters of the patient and normal eye are 5.5 and $4.0 \mathrm{~mm}$, respectively. The sinusoidal lines in red represent the fundamental component of the RETeval TM ERGs, and the black lines indicate the reconstructed flicker ERG waveform, which is the combination of first eight harmonics.

Abbreviation: ERG, electroretinogram.

\section{A}

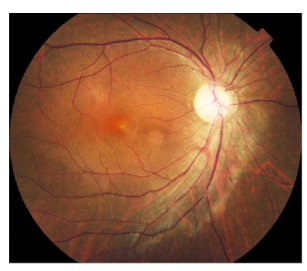

Patient

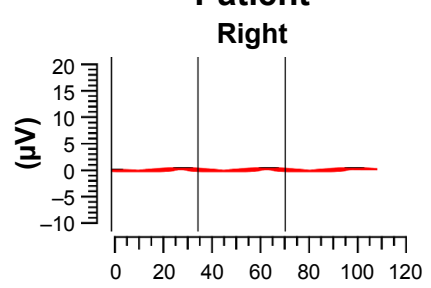

(ms)

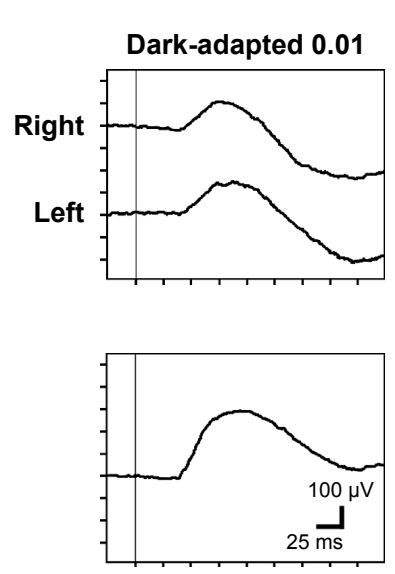

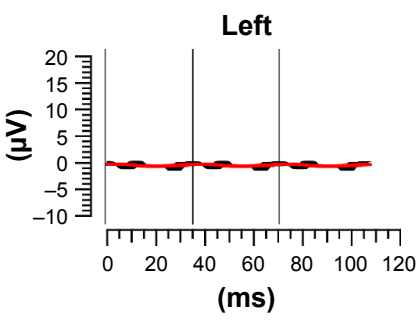

Patient
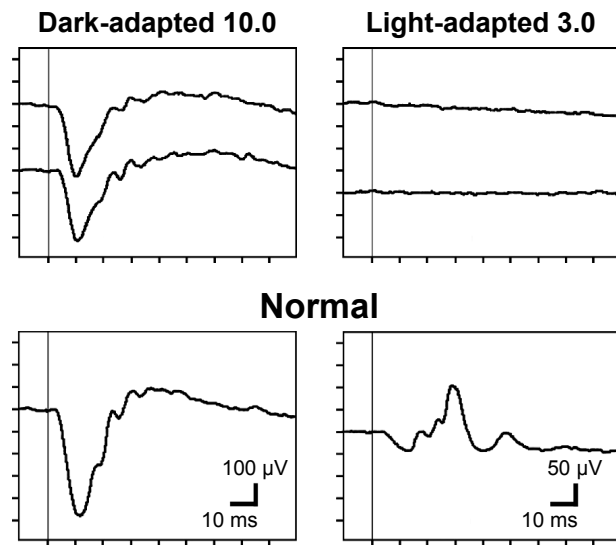
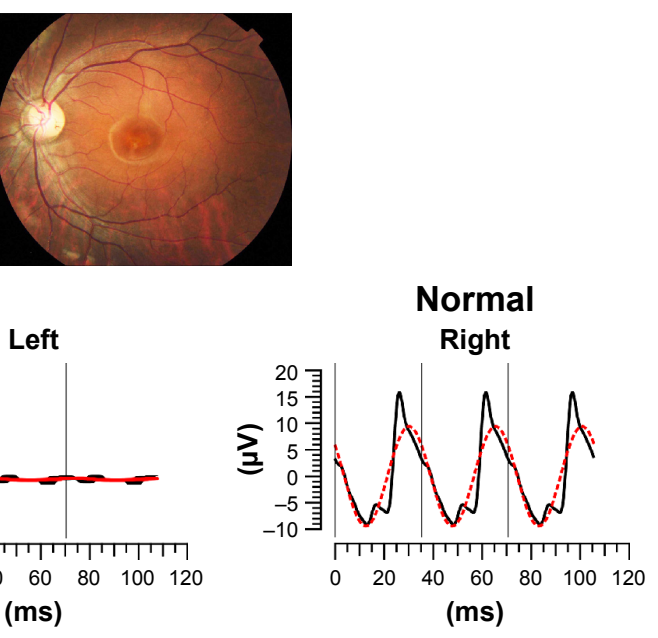

(ms)

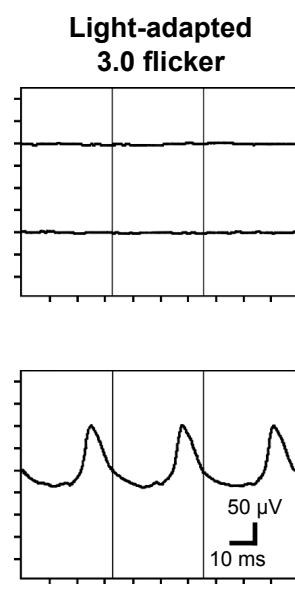

Figure 5 (Continued) 


\section{B}
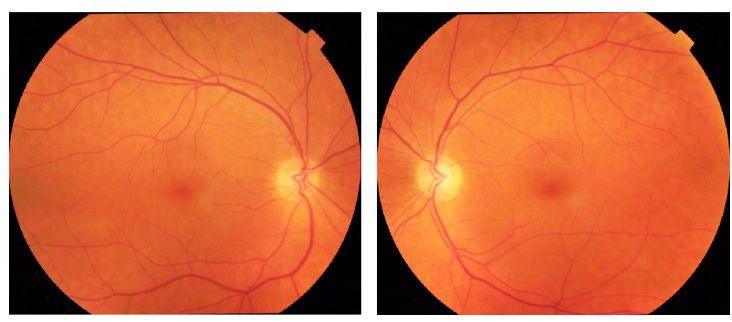

Patient

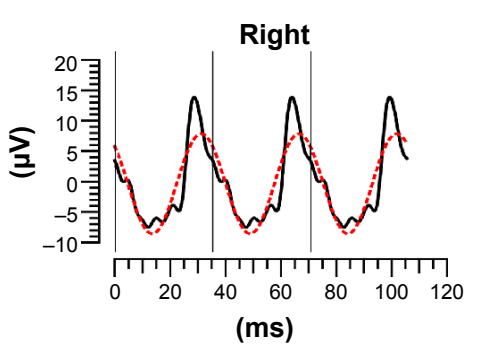

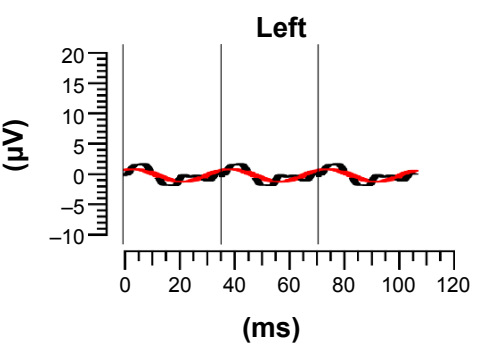

Patient
Dark-adapted 0.01
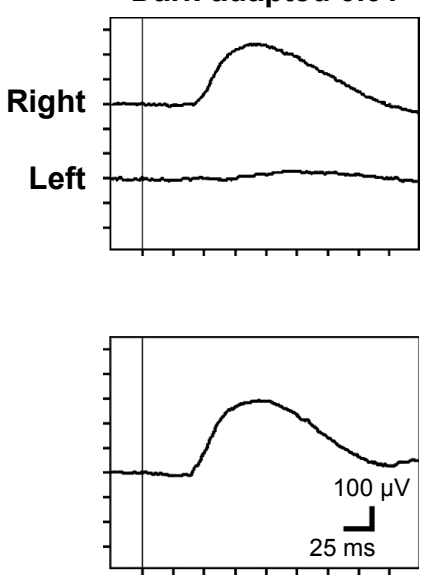

Dark-adapted 10.0

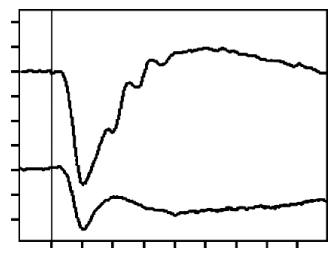

Light-adapted 3.0

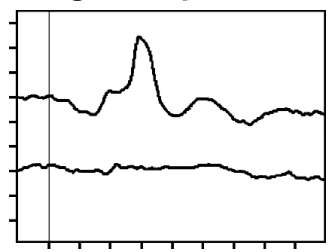

Normal

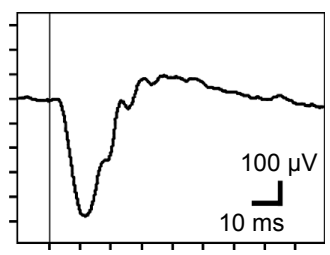

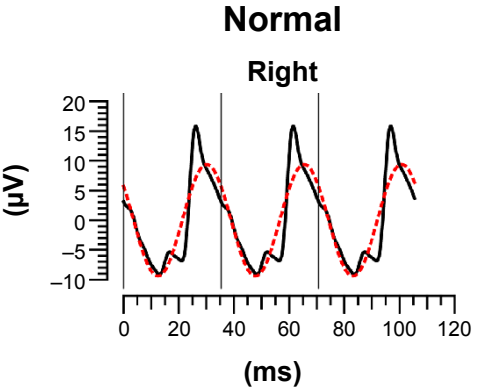

Light-adapted 3.0 flicker
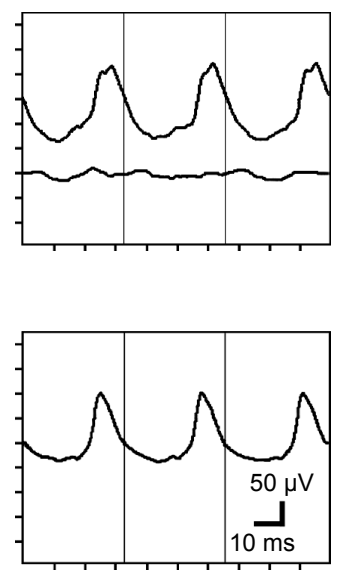

Figure 5 A 23-year-old male with achromatopsia and a 6I-year-old male suspected of autoimmune retinopathy.

Notes: (A) Fundus photographs (upper left), RETeval ${ }^{\text {TM }}$ ERGs (upper right), and conventional full-field ERGs (middle and bottom) of a 23-year-old male diagnosed with achromatopsia. (B) Fundus photographs (upper left), RETeval TM ERGs (upper right), and conventional full-field ERGs (middle and bottom) of a $6 \mathrm{I}$-year-old male who was suspected of having autoimmune retinopathy. In both cases, cone dysfunction of the affected eyes was detected by the RETeval ${ }^{\mathrm{TM}}$ ERGs. The normal examples of the RETeval ${ }^{\mathrm{TM}}$ ERGs were taken from a 35-year-old female. The pupil diameters of the patients in (A), (B), and normal eye are 5.5, 3.5, and 4.5 mm, respectively. The sinusoidal lines in red represent the fundamental component of the RETeval ${ }^{\mathrm{TM}}$ ERGs, and the black lines indicate the reconstructed flicker ERG waveform, which is the combination of first eight harmonics. Abbreviation: ERGs, electroretinograms.

since infancy. His visual acuity was $20 / 125$ both eyes, and ophthalmoscopic examination showed slight atrophic changes in the macula (Figure 5A, upper left). The RETeval ${ }^{\mathrm{TM}}$ recordings showed that the flicker ERGs were extinguished in both eyes (Figure 5A, upper right). The full-field ERGs with the contact lens electrode indicated generalized cone dysfunction and preserved rod function (Figure 5A, middle and bottom), and this patient was diagnosed with achromatopsia.

The ERGs in Figure 5B are from a 61-year-old Caucasian male who developed photophobia in his left eye at the age of 55 years. His visual acuity was $20 / 16$ OU, and ophthalmoscopic examinations revealed no apparent abnormality in both eyes (Figure 5B, upper left). The RETeval ${ }^{\mathrm{TM}}$ showed an almost extinguished flicker response in the left eye (Figure 5B, upper right), and the full-field ERGs with the contact lens electrode revealed generalized cone-rod dysfunction only in his left eye (Figure 5B, middle and bottom). This patient was further investigated for paraneoplastic and autoimmune retinopathies. The results of these two cases indicate that the information obtained by RETeval ${ }^{\mathrm{TM}}$ without pupil dilation reflects an important aspect of retinal dysfunction and useful for judging whether or not to proceed to more detailed electrophysiological tests.

\section{Discussion}

Our results showed that the flicker ERGs recorded by the handheld RETeval ${ }^{\mathrm{TM}}$ device were reduced in eyes with 
various types of cone dysfunction (Figures 1 and 2; Table 1). This device does not require pupil dilation or a contact lens electrode and flicker responses can be recorded even in a consultation room. These results indicate that the RETeval ${ }^{\mathrm{TM}}$ device can be used to screen for cone dysfunction.

In the patients tested, the RETeval ${ }^{\mathrm{TM}}$ ERGs were significantly smaller in diseased eyes than in healthy eyes with almost $100 \%$ accuracy. The time for entire examination is short taking only a few minutes and is better accepted by patients as one patient refused the conventional ERG test but accepted the RETeval ${ }^{\text {TM }}$ test (Figure 4).

However, there are limitations of this device for the screening of retinal dysfunctions. First, the measurement of the flicker ERGs by the RETeval ${ }^{\mathrm{TM}}$ does not provide any information about scotopic function. Nevertheless, the flicker ERG test using RETeval ${ }^{\mathrm{TM}}$ can be used to determine, which patients require additional full-field ERG testing under both scotopic and photopic conditions with dilated pupils. For the cases whose cone dysfunction was detected by the RETeval $^{\mathrm{TM}}$, the latest version of this device is embedded with stimulus protocols for both photopic and scotopic conditions in accordance with the International Society for Clinical Electrophysiology of Vision standard protocol ${ }^{1}$ (Figure 6). The subject in Figure 6 was a 5-year-old boy with night blindness who was once tested with the RETeval ${ }^{\mathrm{TM}}$ at the age of 4 (Figure 4). ERG responses were almost extinguished for both scotopic and photopic conditions, and we confirmed that this patient had retinitis pigmentosa. However, the extended protocol for RETeval ${ }^{\mathrm{TM}}$ takes almost 40 minutes, including time for dark- and light-adaptations and requires a dilation of the pupils unlike the few minutes required to complete a flicker test. Nevertheless, this extended protocol can be considered as one of the useful options when detailed ERG tests are required in children.

A second limitation was that we could not set the retinal illuminance constant during the recordings for the recording from patients with cone dysfunction. For flicker stimulation, RETeval ${ }^{\mathrm{TM}}$ has two stimulating protocols, constant-luminance and constant-troland modes. For subjects without pupil dilation, measurement in the constant-troland is recommended where the flash strength is automatically adjusted in accordance with pupil diameter so that the retinal illuminance is constant irrespective of the pupil diameter. ${ }^{17}$ However, we used the luminance-fixed mode with stimulus strength at $3.0 \mathrm{~cd} \cdot \mathrm{s} / \mathrm{m}^{2}$. We selected this because measurements with the constant-troland mode take twice longer than in the constant-luminance mode. More practically, the measurements in troland can be automatically halted when
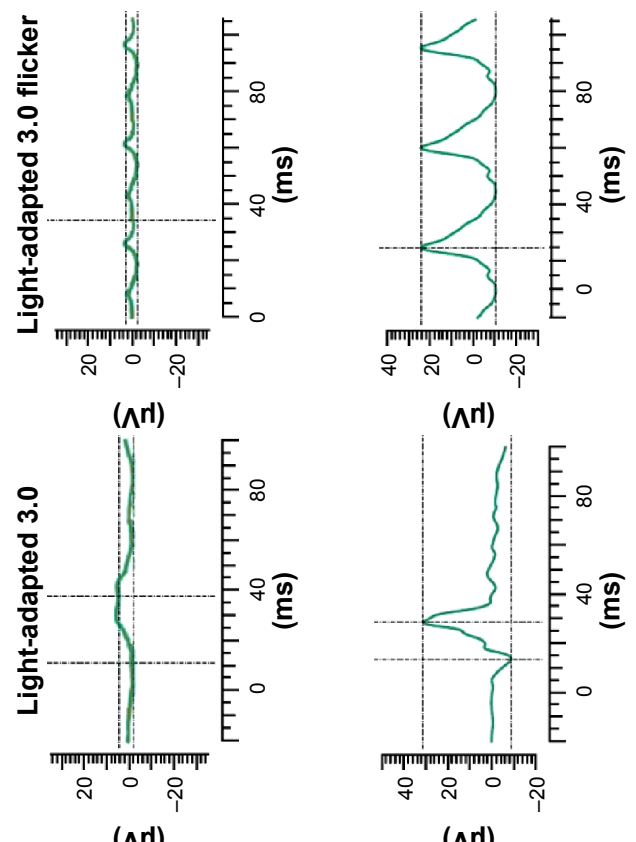

ֻृ

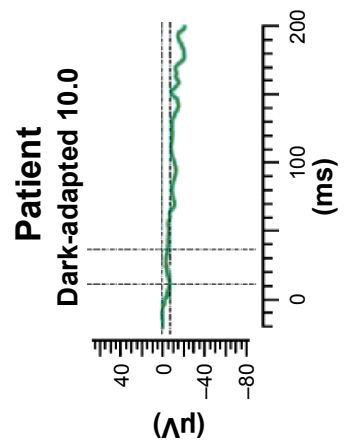

$(\Lambda \mathrm{r})$
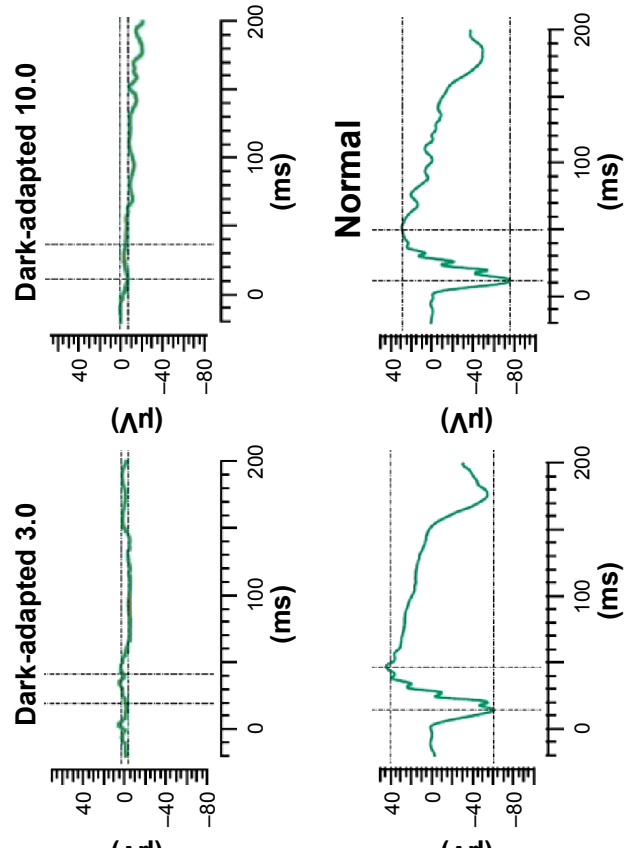

$(\Lambda \mathrm{n})$

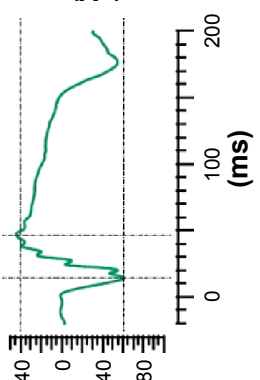

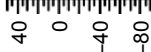

$(\Lambda \mathrm{n})$
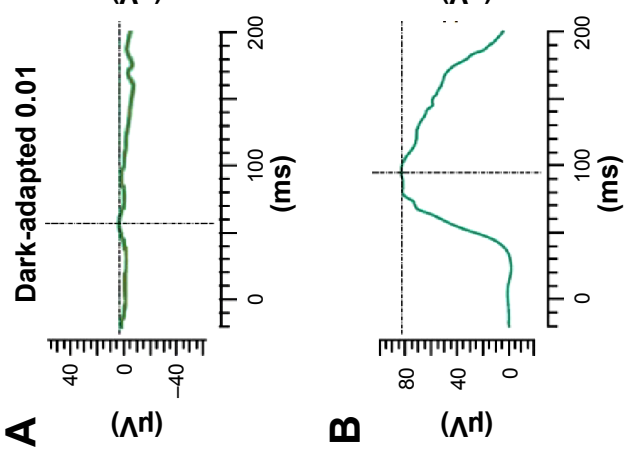

m

㐫

$\frac{d}{n}$ 
the subject blinks and pupil is lost during the recordings. Measurement in the constant-troland mode is thus impractical for children or patients with photophobia who are likely to blink during the recordings.

The problem of the recordings with constant luminance is that the amplitudes and implicit times depend on the pupil diameter: the larger the pupil, the larger the amplitude and shorter the implicit time. ${ }^{22}$ The pupil diameter of the controls $(n=50)$ was significantly correlated with both the amplitude ( $r=0.43, P=0.002$; Pearson correlation coefficient) and implicit time ( $r=-0.386, P=0.006$; Pearson correlation coefficient) (Figure S2). However, we suggest that the variances of the amplitudes and implicit times due to the difference in pupil size did not affect the purpose of our study because 1) we recruited patients and controls whose pupil diameters were between 3.5 and $5.5 \mathrm{~mm}$ to minimize the effect of pupil sizes (Figure S1) and 2) all but one patients had amplitudes lower than the mean $-2.0 \mathrm{SD}$, which cannot be explained by the difference in the pupil size (Figure 1).

We restricted our patients to those who had severe cone dysfunction ascertained by conventional ERG recordings. For practical screening, it would be ideal to detect early stage or mildly affected patients by this device. However, due to the large variations in the amplitudes of normal controls, it will be difficult to screen for mildly affected cone dysfunction by this protocol. Prolonged implicit times are also known to be correlated with reduced cone activities ${ }^{2-4}$; however, the implicit times of RETeval ${ }^{\mathrm{TM}}$ were not significantly correlated with those of the conventional ERGs in our patients. We assume that this discrepancy arose from the fact that in many patients the amplitudes were too low to measure the implicit time accurately. Thus, it will be necessary to examine early stage or mildly affected cone dysfunction with other protocols, that is, constant-luminance mode with data correction in accordance with pupil size or constant-troland mode.

\section{Conclusion}

Our findings indicate that the RETeval ${ }^{\mathrm{TM}}$ system has a potential of being used to screen for cone dysfunction in the clinics where conventional ERG systems are not available. More detailed investigations will be needed to evaluate how the responses obtained by this device are correlated with those by a conventional ERG system and establish protocols to accurately and effectively screen the patients.

\section{Acknowledgments}

This research was supported by 1) research grants from the Ministry of Health, Labor and Welfare, Japan, and
2) Grant-in-Aid for Scientific Research, Japan Society for the Promotion of Science, Japan. The authors have no financial conflict of interest.

\section{Author contributions}

All authors contributed toward data analysis, drafting and revising the paper and agree to be accountable for all aspects of the work.

\section{Disclosure}

The authors report no conflicts of interest in this work.

\section{References}

1. McCulloch DL, Marmor MF, Brigell MG, et al. ISCEV standard for fullfield clinical electroretinography (2015 update). Doc Ophthalmol. 2015; 130(1):1-12.

2. Good PA, Searle AE, Campbell S, Crews SJ. Value of the ERG in congenital nystagmus. Br J Ophthalmol. 1989;73(7):512-515.

3. Massof RW, Johnson MA, Sunness JS, Perry C, Finkelstein D. Flicker electroretinogram in retinitis pigmentosa. Doc Ophthalmol. 1986;62(3): 231-245.

4. Hood DC, Birch DG. Abnormalities of the retinal cone system in retinitis pigmentosa. Vision Res. 1996;36(11):1699-1709.

5. Francis PJ, Marinescu A, Fitzke FW, Bird AC, Holder GE. Acute zonal occult outer retinopathy: towards a set of diagnostic criteria. $\mathrm{Br}$ J Ophthalmol. 2005;89(1):70-73.

6. Weleber RG, Watzke RC, Shults WT, et al. Clinical and electrophysiologic characterization of paraneoplastic and autoimmune retinopathies associated with antienolase antibodies. Am J Ophthalmol. 2005; 139(5):780-794.

7. Bresnick GH, Palta M. Temporal aspects of the electroretinogram in diabetic retinopathy. Arch Ophthalmol. 1987;105(5):660-664.

8. Tahara K, Matsuura T, Otori T. Diagnostic evaluation of diabetic retinopathy by $30-\mathrm{Hz}$ flicker electroretinography. Jpn J Ophthalmol. 1993; 37(2):204-210.

9. Johnson MA, Marcus S, Elman MJ, McPhee TJ. Neovascularization in central retinal vein occlusion: electroretinographic findings. Arch Ophthalmol. 1988;106(3):348-352.

10. Larsson J, Andréasson S. Photopic $30 \mathrm{~Hz}$ flicker ERG as a predictor for rubeosis in central retinal vein occlusion. Br J Ophthalmol. 2001;85(6): 683-685.

11. Ponjavic V, Andreasson S. Multifocal ERG and full-field ERG in patients on long-term vigabatrin medication. Doc Ophthalmol. 2001;102(1): 63-72.

12. Zoumalan CI, Zamanian RT, Doyle RL, Marmor MF. ERG evaluation of daily, high-dose sildenafil usage. Doc Ophthalmol. 2009;118(3): 225-231.

13. Fulton AB, Brecelj J, Lorenz B, Moskowitz A, Thompson D, Westall CA. Pediatric clinical visual electrophysiology: a survey of actual practice. Doc Ophthalmol. 2006;113(3):193-204.

14. Fahim AT, Khan NW, Zahid S, et al. Diagnostic fundus autofluorescence patterns in achromatopsia. Am J Ophthalmol. 2013;156(6):1211-1219. e1212.

15. Miyake Y, Tsunoda K. Occult macular dystrophy. Jpn J Ophthalmol. 2015;59(2):71-80.

16. Roosing S, Thiadens AA, Hoyng CB, Klaver CC, den Hollander AI, Cremers FP. Causes and consequences of inherited cone disorders. Prog Retin Eye Res. 2014;42:1-26.

17. Kato K, Kondo M, Sugimoto M, Ikesugi K, Matsubara H. Effect of pupil size on flicker ERGs recorded with RETeval system: New mydriasis-free full-field ERG system. Invest Ophthalmol Vis Sci. 2015; 56(6):3684-3690. 
18. Yasuda S, Kachi S, Ueno S, Piao CH, Terasaki H. Flicker electroretinograms before and after intravitreal ranibizumab injection in eyes with central retinal vein occlusion. Acta Ophthalmol. 2015;93(6): e465-e468.

19. Sieving PA, Arnold EB, Jamison J, Liepa A, Coats C. Submicrovolt flicker electroretinogram: cycle-by-cycle recording of multiple harmonics with statistical estimation of measurement uncertainty. Invest Ophthalmol Vis Sci. 1998;39(8):1462-1469.

20. Severns ML, Johnson MA, Merritt SA. Automated estimation of implicit time and amplitude from the flicker electroretinogram. Appl Opt. 1991; 30(16):2106-2112.
21. McAnany JJ, Nolan PR. Changes in the harmonic components of the flicker electroretinogram during light adaptation. Doc Ophthalmol. 2014; 129(1):1-8.

22. Gagné AM, Lavoie J, Lavoie MP, Sasseville A, Charron MC, Hébert M. Assessing the impact of non-dilating the eye on full-field electroretinogram and standard flash response. Doc Ophthalmol. 2010;121(3):167-175. 


\section{Supplementary materials}
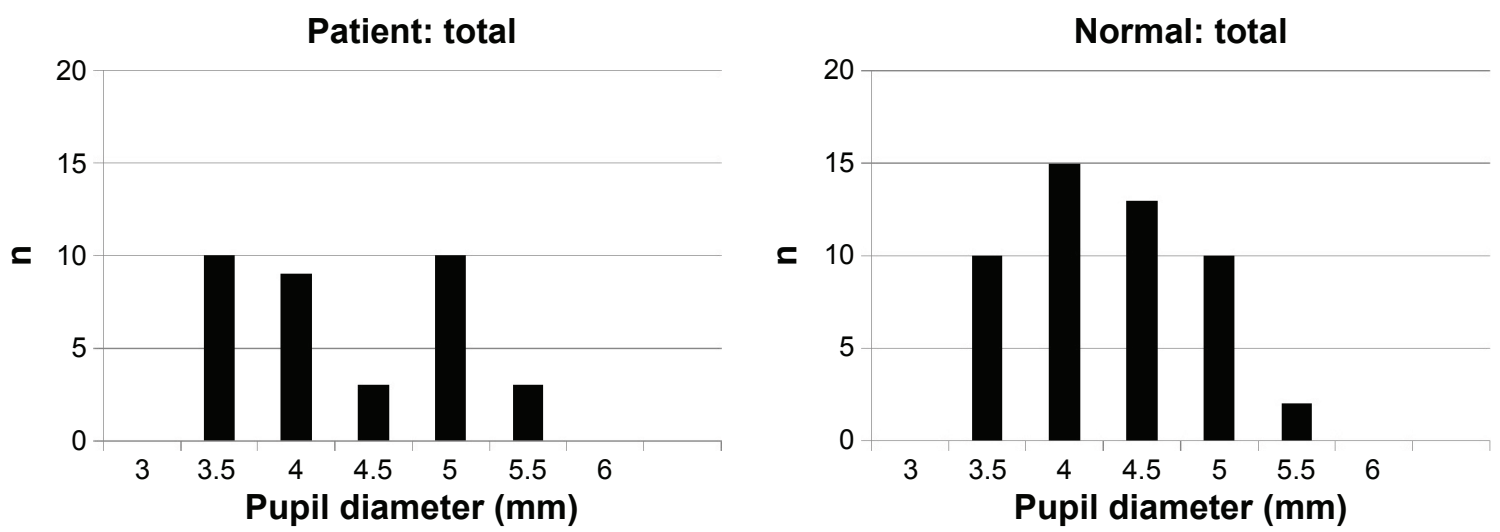

Patient: $\leq \mathbf{2 0}$ years
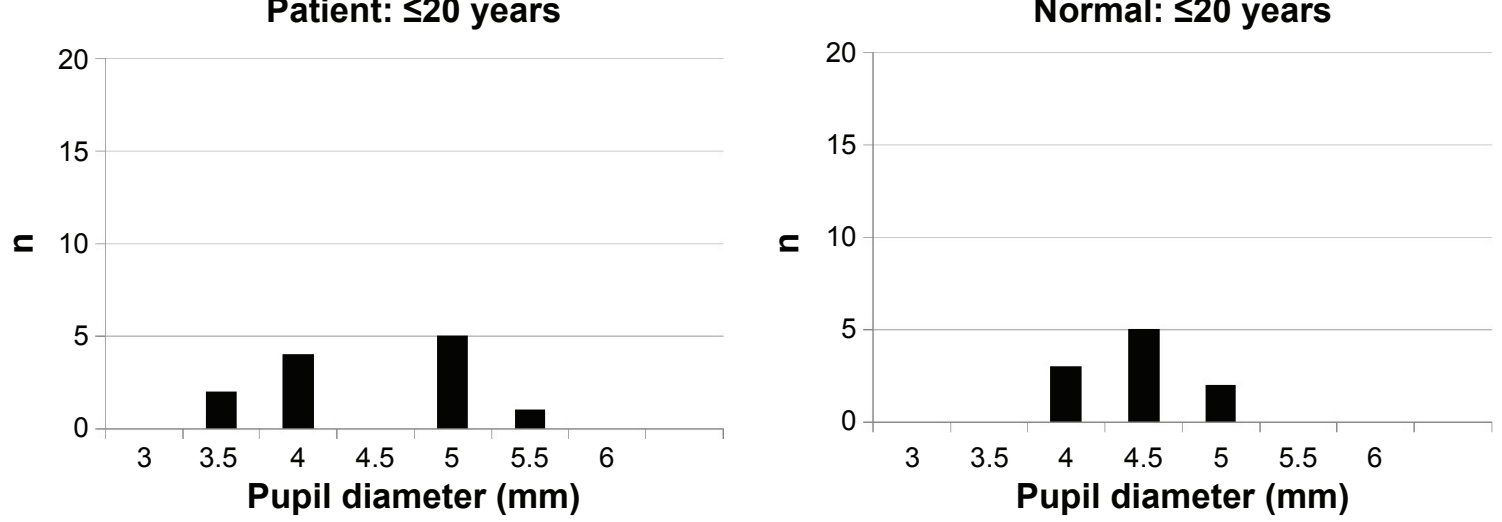

Patient: 21-40 years

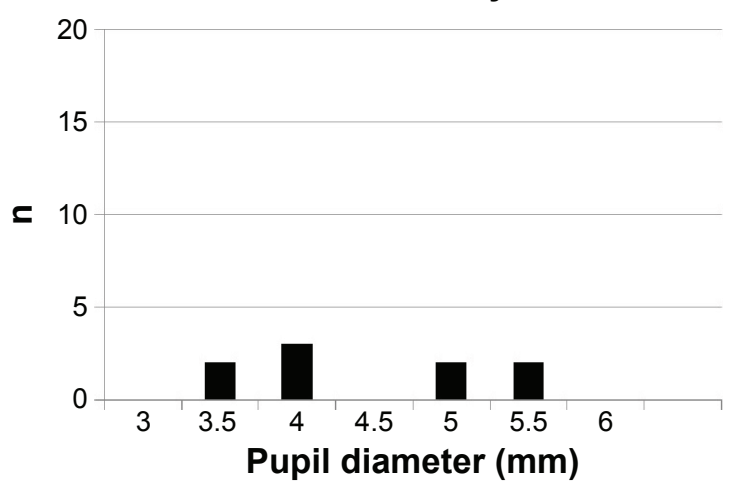

Normal: 21-40 years

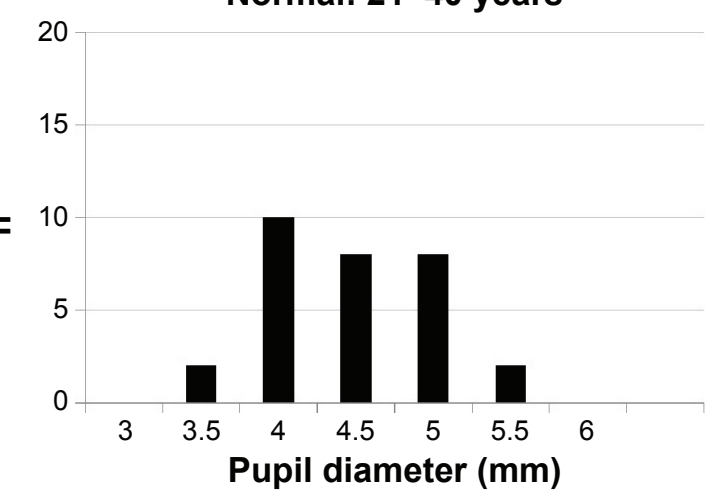

Patient: $\geq 41$ years
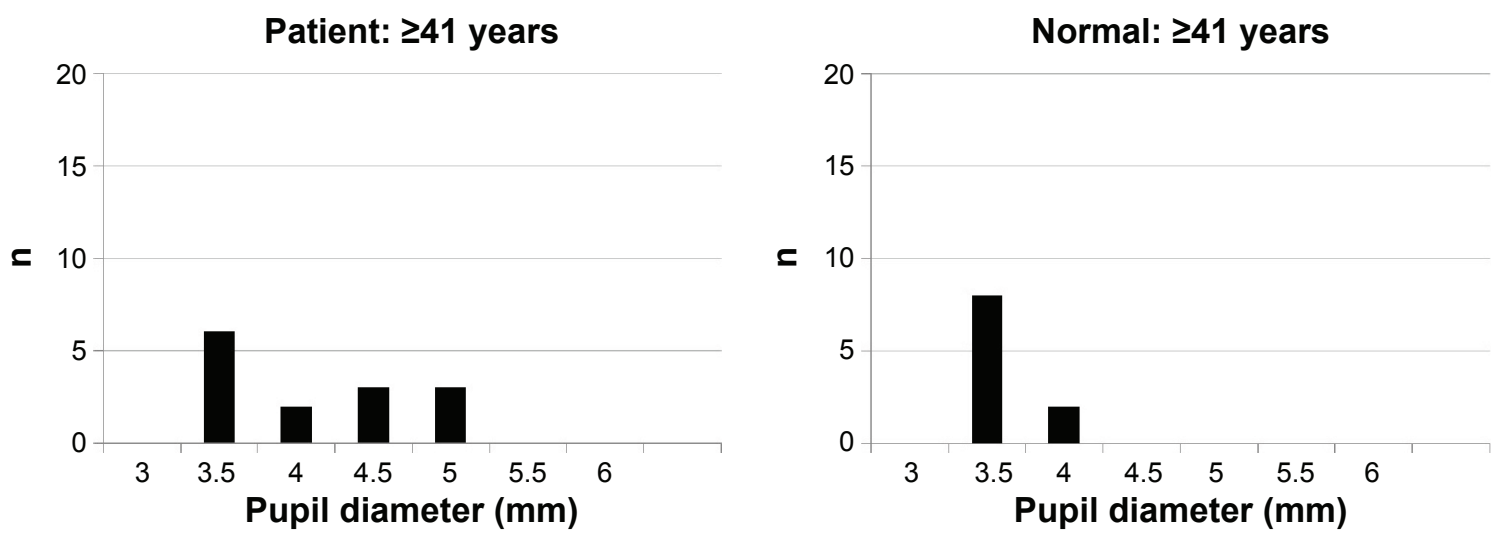

Figure SI Pupil diameters before recording in patients and controls.

Notes: Patients and controls are divided into three age groups: Group A $\leq 20$ years, Group B $21-40$ years, and Group $C \geq 4$ I years. In each group, there was no significant difference in the pupil diameters between patients and controls. 


\section{A Pupil size and amplitude in RETeval ${ }^{\mathrm{TM}}$ (controls, $n=50$ )}

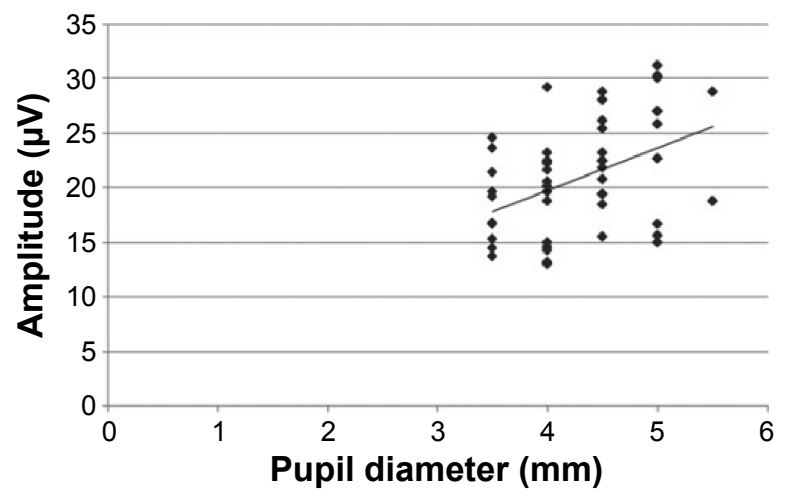

\section{B Pupil size and implicit time in RETeval ${ }^{\mathrm{TM}}$} (controls, $n=50$ )

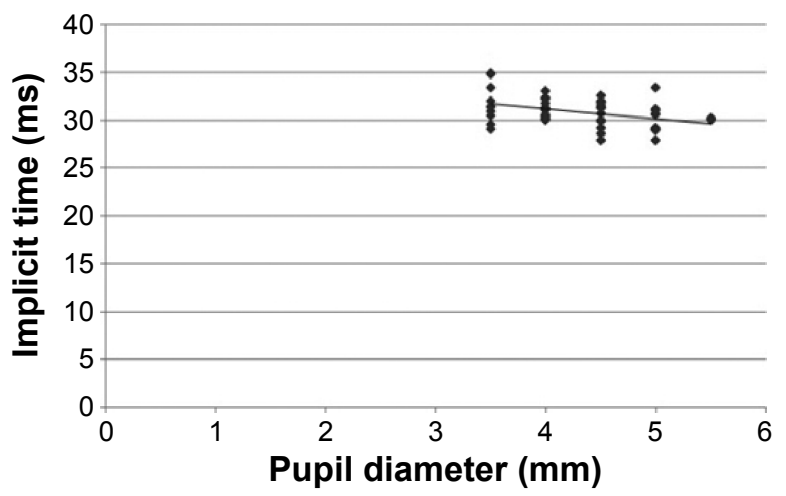

Figure S2 Correlation between pupil diameter and response of the RETeval ${ }^{\mathrm{TM}}$ in control subjects.

Notes: (A) Amplitude; (B) implicit time. The pupil diameters of the controls $(n=50)$ were significantly correlated with both the amplitude (left, $r=0.43, P=0.002 ;$ Pearson correlation coefficient) and implicit time (right, $r=-0.386, P=0.006$; Pearson correlation coefficient).

Abbreviation: $n$, number.

\section{Publish your work in this journal}

Clinical Ophthalmology is an international, peer-reviewed journal covering all subspecialties within ophthalmology. Key topics include: Optometry; Visual science; Pharmacology and drug therapy in eye diseases; Basic Sciences; Primary and Secondary eye care; Patient Safety and Quality of Care Improvements. This journal is indexed on
PubMed Central and CAS, and is the official journal of The Society of Clinical Ophthalmology (SCO). The manuscript management system is completely online and includes a very quick and fair peer-review system, which is all easy to use. Visit http://www.dovepress.com/ testimonials.php to read real quotes from published authors. 